\title{
El Instituto Superior de Música. Una historia en varios movimientos
}

Hugo Druetta ${ }^{(1)}$

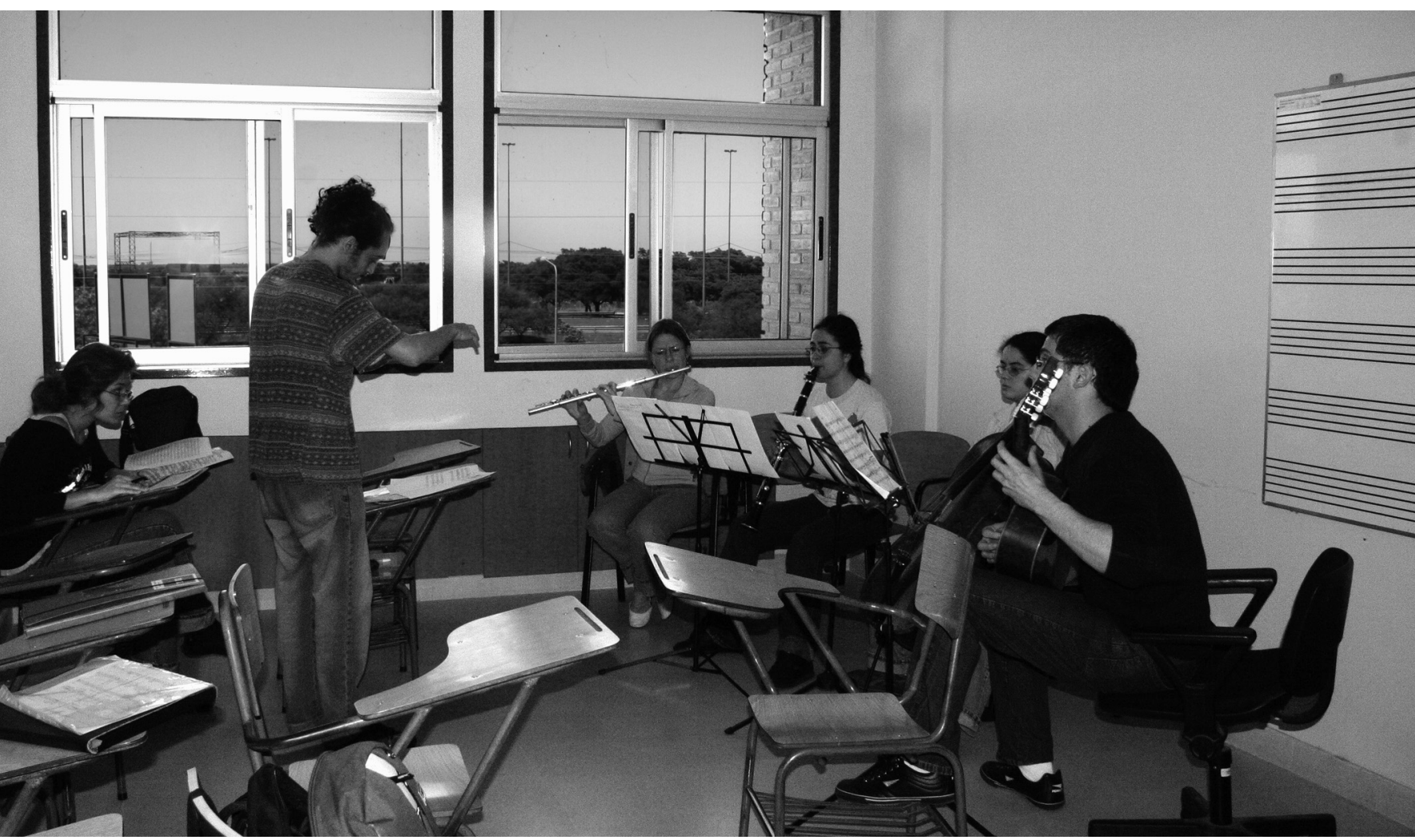

(1) Director del ISM-FHUC-UNL 
El actual Instituto Superior de Música, fue creado por Resolución № 534 del 13 de octubre de 1947, dictada por el entonces Interventor de la Universidad Nacional del Litoral Dr. Edgardo M. Hillaire Chaneton.

Entre los considerandos de la Resolución de creación, de la inicialmente denominada, Escuela Superior de Música y Canto, podemos leer:

"Que corresponde a los centros de cultura superior, en ejercicio de su elevada función educadora, propender a que las manifestaciones del espíritu se desarrollen y cultiven con el mismo noble afán con que desde la cátedra y el laboratorio se plasman y difunden los conocimientos de la técnica y de la ciencia en sus más diversas disciplinas; (...) Que la ciudad de Santa Fe ofrece promisorias perspectivas para la creación de una escuela superior de música y canto por ser el punto de influencia de un vasto sector del litoral cuya juventud ya ha demostrado su inclinación por el arte; (...) Que debido a la falta en esta zona del país, de institutos de la jerarquía del que se trata, son múltiples las dificultades que se les presentan a los que, siguiendo un impulso vocacional, anhelan perfeccionar sus conocimientos, pues, para lograr ese objetivo, tienen necesariamente que alejarse del medio en que viven"

Los motivos que llevaron, a que un inquieto grupo de personas preocupadas por la realidad artística de aquel entonces, se movilizara en pos de la creación de un espacio universitario destinado a la formación artística, aparecen con toda claridad en palabras de su primer Delegado Organizador, Horacio Caillet-Bois, en un artículo escrito para el Cuaderno $N^{0} 1$, del Departamento de Musicología de la Escuela Superior de Música de la Universidad Nacional del Litoral, en el año 1949:

"El gusto por la música es tan grande y su apetencia tan urgente aquí como allá [el autor compara Europa y Estados Unidos con nuestro país]. Pero aquí esa necesidad popular no puede saciarse sino a medias, y sólo en los grandes 
centros de población, por falta de conjuntos orquestales que llenen esa misión. Hay que correr sin tardanza a salvar esa falla de nuestra cultura artística. Para ello no hay otro camino que el de preparar a las nuevas generaciones, sólidamente, en el gusto y en el ejercicio de la música. De allí saldrá todo lo demás. La creación de la Escuela Superior de Música de la Universidad Nacional del Litoral, es un paso avanzado en esta cruzada. Los resultados no tardarán en dejarse sentir en toda esta región del Litoral. Puede decirse que se notan, a pesar de que apenas tiene un año de existencia. Por lo pronto la inquietud despertada en una entusiasta promoción de alumnos, que frecuentan sus aulas, el interés concitado por la enseñanza y el conocimiento de todos los instrumentos fundamentales -no solamente el piano y el violín-; la creación de cátedras formativas que despiertan en el alumno el gusto por la investigación y los problemas filosóficos y estéticos conexos con esta disciplina, constituyen un panorama nuevo en nuestra vida intelectual, artística, universitaria, y anticipan una corriente regional que se definirá algún día en la música nativa."

Para darnos una idea de la trascendencia que tuvo en la sociedad santafesina, la creación de la Escuela Superior de Música y Canto, transcribimos la opinión de la Prof. Amalia Marta Pérez quien, en su libro "La Música en Santa Fe" nos dice al respecto:

"Al nacer, en la década del cuarenta, el Instituto Superior de Música de la Universidad Nacional del Litoral marca un hito de trascendencia en la vida musical de Santa Fe y constituye un signo del grado de madurez alcanzado por ésta en su vida cultural.

Desde el momento en que nace a la vida casi al promediar el siglo, su aporte a la práctica musical alcanzará relieves inestimables con períodos de verdadero apogeo, dando a conocer ciclo integrales de obras capitales del repertorio musical universal, ofreciendo muchas primeras audiciones absolutas para Santa Fe, reeditando las de innumerables obras consagradas y, esencialmente, cumpliendo con su objetivo primero y último, cual es la formación del músico profesional Ilamado a cubrir necesidades crecientes en los planos tanto artísticos como docentes."

La Escuela Superior de Música y Canto que, a partir de 1960 pasa a denominarse Instituto Superior de Música, ha sido desde sus inicios un claro referente de la cultura musical de esta región. 
A partir de la información contenida en documentos oficiales y periodísticos, se puede dimensionar la fuerte tarea de extensión que siempre acompañó a las actividades formativas tanto en lo artístico como en la docencia. Jornadas, encuentros, conciertos, audiciones, publicaciones han ocupado la atención de las diferentes gestiones que se sucedieron en el tiempo.

Lamentablemente, muchas de las buenas intenciones que se observan como enunciados o iniciativas de algún período, se encuentran discontinuadas en otro, lo que de alguna manera refleja un generalizado panorama de interrupciones institucionales que, producidas a nivel nacional, modificaban las gestiones y las acciones en el Instituto.

La fecha de iniciación de las actividades académicas oficiales del ISM fue el 6 de mayo de 1948, mediante un concierto que a modo de evento inaugural, se llevó a cabo en el Paraninfo de la UNL. Ésta es la fecha que se ha tomado como referencia para contabilizar los años de vida de esta institución que festejará su sexagésimo aniversario en el año 2008.

En estos casi 60 años de vida, bajo diferentes denominaciones de cargos, sus autoridades, ordenadas cronológicamente, han sido:

- Horacio Caillet-Bois

- José Berrini

- Juan Zocchi

- Roberto Locatelli

- Sebastián Lombardo

- Roberto Locatelli

- Sigfrido Prager

- Eduardo Barbagelata

- Juan Pedro Franze

- Emilio Dublanc

- Elisa del Carmen Meyer de Zurbriggen

- Ana Rosa Calderón

- Guillermo Bonet Müller

- Héctor Ariel Nardi

- Nelly Del Curto

- Jorge Molina

- Enrique Núñez

- Mariano Cabral Migno 
El Instituto Superior de Música, orientado en sus inicios principalmente a la formación de Intérpretes Musicales, fue incorporando progresivamente y mediante diferentes instancias académicas, la formación de compositores, directores (de coro y de orquesta), docentes especializados y de educación musical general e investigadores.

El Consejo Superior de la UNL mediante Resolución No 189 del año 1995, resuelve la Integración del ISM a la entonces Facultad de Formación Docente en Ciencias (hoy Facultad de Humanidades y Ciencias), la cual tomó efecto a partir del primero de enero de 1996.

Durante estos últimos once años, se ha iniciado la progresiva normalización de la planta docente mediante concursos ordinarios, así como también, se han ensayado, mediante diversas iniciativas conjuntas, los diseños tentativos para una convivencia institucional que, por un lado encuadren estatutariamente la situación del ISM en el contexto general de la UNL y por otra parte, garanticen la permanencia de los rasgos idiosincrásicos de una comunidad orgullosa de su historia y conciente del aporte que sus logros artísticos, académicos y científicos le han reportado a la UNL desde un principio y a la propia Facultad de Humanidades y Ciencias en los últimos tiempos.

Como parte del reconocimiento a los mismos, no podemos dejar de mencionar la construcción del nuevo edificio, cuya inauguración llevada a cabo el 9 de junio de 2006, marcó un hito de gran trascendencia en la vida Institucional por cuanto significó, la inserción definitiva en un contexto universitario constituido en punto de partida para nuevas iniciativas.

En este sentido, el nuevo enclave del ISM y las innumerables opciones de interacción con la estructura universitaria en general y con la de la Facultad de Humanidades y Ciencias en particular, nos permiten apuntalar las tareas académicas, de extensión y de investigación que se venían desarrollando y plantearnos nuevas metas.

Hoy, el Instituto Superior de Música cuenta con las siguientes carreras:

- Tecnicatura en composición musical con medios electroacústicos

- Profesorado de Música

- Profesorado de Música con Orientaciones en (canto, piano, guitarra, violín, violoncello, flauta, clarinete, saxofón, percusión, dirección orquestal, dirección coral y composición) 
- Licenciaturas en Música con Orientaciones en (canto, piano, guitarra, violín, violoncello, flauta, clarinete, saxofón, percusión, dirección orquestal, dirección coral y composición)

- Licenciatura en Teoría y Crítica de la Música (ciclo de licenciatura dictada bajo la modalidad a distancia)

- Licenciatura en Sonorización y Grabación, en trámite de aprobación

- Carrera de musicoterapia, en etapa de diseño

Además de su cuerpo de Profesores, el ISM cuenta con una Orquesta de Cámara, un Coro de Cámara y el Estudio de Fonología y Música Electroacústica, que garantizan las condiciones de estudio y prácticas requeridas por las diferentes especialidades.

En cuanto a la investigación, desde hace ya varios años, hay grupos de investigadores que llevan adelante proyectos CAI+D así como también una constante adhesión de los estudiantes a los programas de iniciación a la investigación. Las actividades de extensión se han ido incrementando conforme el nuevo espacio edilicio lo ha permitido. Como muestra de lo realizado en el corriente año, podemos mencionar:

- Encuentro de Investigadores sobre Rock

- 5tas. Jornadas Santafesinas de Música Contemporánea (Co-organizadas con la Dirección de Cultura de la UNL)

- Jornadas pre-congreso Nacional de Musicoterapia

- El primer congreso de educación musical denominado: Músicos en Congreso, puntos de llegada y puntos de partida en la educación musical.

- Ciclo de conciertos "ISM en el Paraninfo"

- 8va edición de Musicalia

- Innumerables audiciones y muestras de cátedras, cursos, charlas y clases magistrales.

Actualmente, el ISM orienta sus fuerzas a consolidar su lugar como centro de referencia en lo que a producción- difusión respecta, mediante la continuidad de la revista y la incorporación de nuevas publicaciones que retoman la edición tanto de música escrita, como de registro discográfico. 
Por último cabe mencionar que, una institución de formación musical, cumple variadas e imprescindibles funciones. Una de ellas es la de constituirse en ámbito de preservación de las disciplinas sobre las que basa sus actividades. En este sentido el ISM, conserva los repertorios consagrados de la tradición musical occidental, resguarda los espacios para el estudio de las músicas de distintas culturas y tiempos y ofrece un espacio para el estudio, creación y difusión de aquellas expresiones que no cuentan con la inserción en los esquemas de difusión más convencionales y masivos.

Como anhelo institucional, devenido en desafío, se pueden mencionar el proyecto en diseño de una carrera de posgrado, y las modificaciones de los Planes de estudio, necesarias para dar respuesta a las nuevas demandas de la actual configuración socio educativa de nuestra región y facilitar la generación de nuevas carreras.

En una realidad cambiante y compleja, con áreas de conocimiento que se redefinen prácticamente sin solución de continuidad, el Instituto Superior de Música se encuentra inmerso en un panorama dinámico, con estructuras preparadas para la canalización de las más diversas propuestas, y tal vez éste sea el mejor respaldo que nos ofrece la Universidad Nacional del Litoral a través de la Facultad de Humanidades y Ciencias, la posibilidad de seguir soñando a la par de nuestros antecesores.

\section{Referencias bibliográficas.}

Pérez Chiara, Amalia Marta (1973): La música en Santa Fe, separata de la Historia de las Instituciones de la Provincia de Santa Fe, Tomo 5, $2^{a}$ parte; El siglo XX, IX. La música en el ámbito universitario. El Instituto de Música.

Flury, Lázaro (1980): Reseña histórica del Instituto Superior de Música de la Universidad Nacional del Litoral, sin datos editoriales, Santa Fe.

Molina, Jorge (1990): La música contemporánea en Santa Fe, en la Revista del Instituto Superior de Mú- sica de la UNL No 2, Centro de Publicaciones de la UNL, Santa Fe.

Blumberg, Edgardo (1999): Sobre el Instituto Superior de Música, incluido en la Revista del Instituto Superior de Música de la UNL № 6, Centro de Publicaciones de la UNL, Santa Fe.

Pínnola, Fabián (...): Informe del Proyecto El Cantar de la Memoria. La Historia del ISM.

Documentos institucionales archivados en el ISM. 